\title{
Sexual transmission of HIV in Africa
}

\section{Other routes of infection are not the dominant contributor to the African epidemic.}

T he human immunodeficiency virus (HIV) epidemic in sub-Saharan Africa continues to grow at an alarming rate, with 3.5 million people infected last year alone $^{1}$. It has recently been proposed that heterosexual transmission cannot account for more than $35 \%$ of HIV incidence in this region, and that parenteral transmission, probably through unsterile medical practices, is the main route of infection ${ }^{2}$. A key prediction of this hypothesis is that the epidemic history of HIV in sub-Saharan Africa is similar to that of other blood-borne pathogens. To test this, we compared prevalence estimates of HIV with those of hepatitis $\mathrm{C}$ virus $(\mathrm{HCV})$, which has a far greater rate of parenteral than sexual transmission. We show that HIV and HCV have such different epidemic histories in sub-Saharan Africa that parenteral transmission is unlikely to be the main source of HIV infection.

We plotted comparable estimates of HIV and HCV prevalence in the general population for all countries in the world for which data are available (Fig. 1a). Countries are segregated according to whether their $\mathrm{HCV} / \mathrm{HIV}$ prevalence ratio is greater or less than 1 (black line in Fig. 1a). This distinction is very conservative, given that $\mathrm{HCV}$ is about six times more infectious than HIV by parenteral transmission ${ }^{3}$. Most countries have a ratio of $>1$, as expected from the higher global prevalence of HCV. However, countries with ratios of $<1$ are largely from sub-Saharan Africa (Fig. 1a, red circles) and, crucially, these contain the majority of HIV-infected individuals worldwide (as indicated by circle sizes in Fig. 1a; India is the exception). This observation is contrary to what would be expected if most cases of HIV infection were attributable to parenteral transmission. It cannot be argued that HCV was absent from sub-Saharan Africa at the onset of the HIV pandemic, because HCV genotypes 1, 2 and 4 have been endemic to west and central Africa for several centuries, and subtype $5 \mathrm{a}$ has been present in South Africa for at least 50 years ${ }^{4,5}$.

To complement this cross-sectional prevalence analysis, we plotted the temporal change in HIV and HCV prevalence in South Africa, the country with the greatest number of HIVinfected individuals in Africa, and for which annual data are available (Fig. 1b). Crucially, HCV and HIV were at similarly low prevalences at the beginning of the 1990s. Since then, the increase in HIV prevalence among adults has been swift, rising from $0.7 \%$ in 1990 to $24.5 \%$ in 2000 (ref. 6), whereas estimates of $\mathrm{HCV}$ prevalence have remained relatively low over the same period (for data

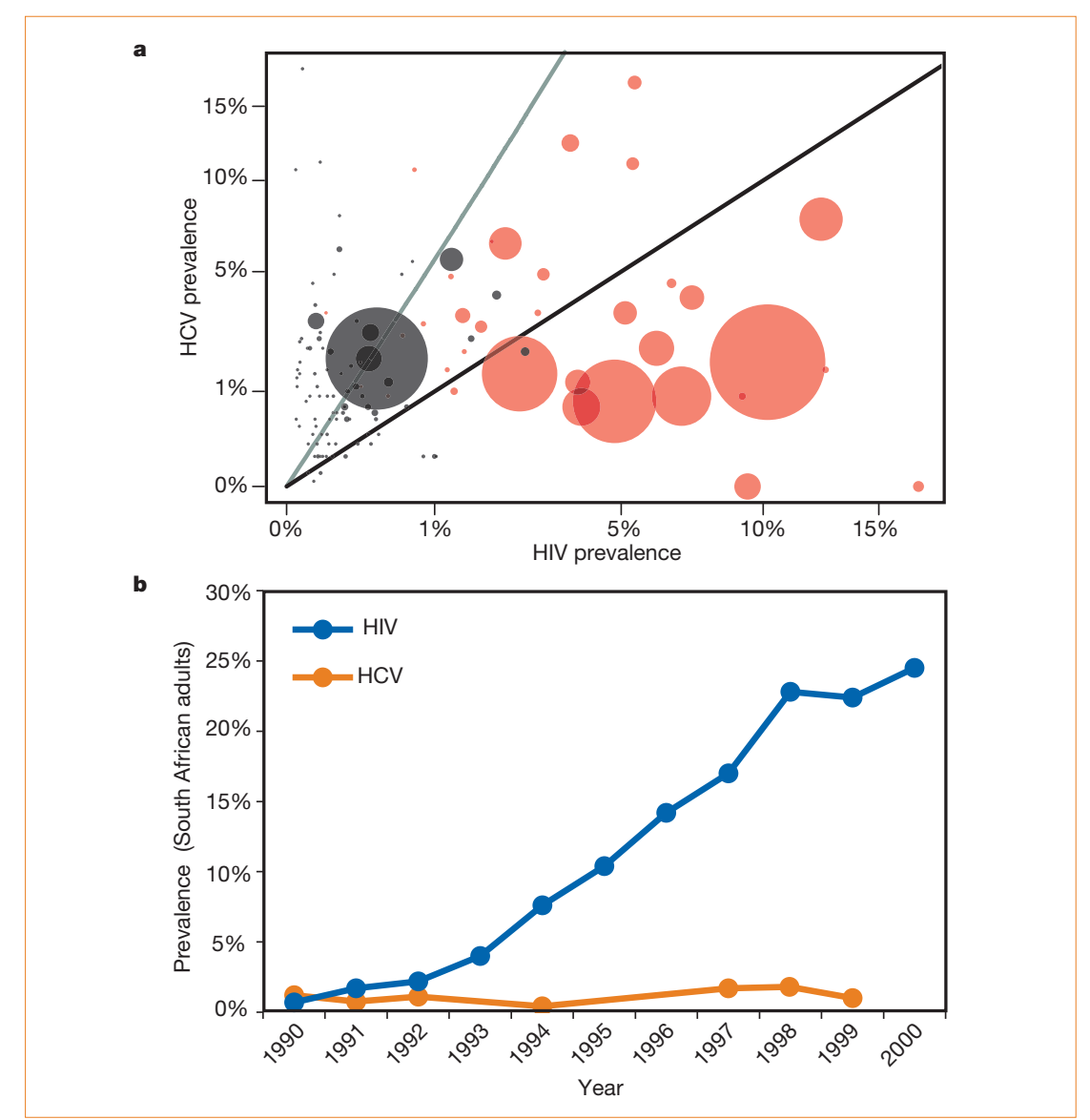

Figure 1 Comparison of epidemic histories of human immunodeficiency virus (HIV) and hepatitis C virus (HCV) in different countries, and in South Africa during the 1990s. a, HCV and HIV prevalence in the general population (including children) of every country for which data are available. The arcsine square-root transformation for proportions was used because the original distribution of points was strongly L-shaped. Sub-Saharan African countries are represented by red circles; other countries are represented by black circles; the radius of each circle is proportional to the number of HIV infected individuals in that country. Countries below the black line have a higher prevalence of HIV than HCV; the opposite is true for countries above the black line. The grey line represents an HCV:HIV prevalence ratio of 6 , which indicates the relative parenteral transmissibility of the two pathogens ${ }^{3}$. All prevalence figures apart from five HCV values were obtained from World Health Organization sources. b, HIV and HCV prevalence in South African adults from 1990 to 2000 (estimates for HIV from ref. 6; estimates for HCV from sources listed in supplementary information).

sources, see supplementary information). The absence of a comparable increase in the prevalence of $\mathrm{HCV}$, a virus that is more easily transmitted parenterally, suggests that current levels of HIV prevalence are not primarily the result of using unsterile medical equipment or contaminated blood products.

Although it is important to highlight the issue of pathogen transmission by unsafe injection, the different epidemic histories of HIV and HCV in sub-Saharan Africa indicate that parenteral transmission is not the dominant contributor to the African HIV epidemic. Consequently, the introduction of widespread drug treatment for HIV and cofactor infections, as well as continued efforts to encourage safe sex, are urgently required to reduce the spread of HIV in Africa.

Polly R. Walker, Michael Worobey, Andrew Rambaut, Edward C. Holmes, Oliver G. Pybus Department of Zoology, University of Oxford, South Parks Road, Oxford OX1 3PS, UK e-mail:edward.holmes@zoo.ox.ac.uk

1. UNAIDS. Report on the Global HIV/AIDS Epidemic 2002 (2002). 2. Gisselquist, D. \& Potterat, J. J. Int. J. STD AIDS 14, 162-173 (2003).

3. Goldmann, D. A. J. Allergy Clin. Immunol. 110, S21-S26 (2002). 4. Smith, D. B. et al. J. Gen. Virol. 78, 321-328 (1997).

5. Pybus, O. G. et al. Science 292, 2323-2325 (2001).

6. South African Department of Health. National HIV and Syphilis Sero-prevalence Survey of Women Attending Public Antenatal Clinics in South Africa 2000 (2001).

Supplementary information accompanies this communication on Nature's website.

Competing financial interests: declared none. 